\title{
Riscos associados ao uso de fármacos psicoativos na população idosa
}

\author{
Risks associated with the use of psychoactive drugs in the elderly population \\ Riesgos asociados al uso de psicofármacos en la población anciana
}

Gabrielle Froz Rodrigues ${ }^{1 *}$, Annelysa Vitória Souza Ramalho², Lívia Fernandes Monteiro da Mata ${ }^{3}$, Maria Eduarda Oliveira Có ${ }^{4}$, Paula Andrade Ferreira ${ }^{5}$, Paula Lima Sperandio ${ }^{6}$, Rafael Rolli Haddad ${ }^{7}$, Raquel Campos Pereira ${ }^{8}$, Samuel Oliveira Dumont Horta ${ }^{3}$, Monica Isaura Corrêa ${ }^{9}$.

\section{RESUMO}

Objetivo: Descrever os riscos do uso de ansiolíticos e indutores do sono na população idosa. Revisão bibliográfica: A polifarmácia cresce cada vez mais dentro da população idosa, em vista disso, o próprio processo de envelhecimento natural gera alterações nas etapas de farmacocinética e farmacodinâmica dos fármacos trazendo, ao idoso, algumas reações adversas. Os medicamentos mais utilizados dentro dessa faixa etária são representados pela classe dos ansiolíticos e indutores do sono. $O$ uso prolongado de benzodiazepínicos, cujo consumo alcança de 15\% a 43\% em adultos com idade de 65 anos ou mais, aumentam o risco de efeitos colaterais, tais como, dependência, sedação, comprometimento cognitivo e psicomotor e quedas. Já os indutores do sono representados, principalmente, pelos antidepressivos tricíclicos, podem gerar efeitos de sedação, hipotensão postural, constipação e demência nos idosos, devido a sua alta ação anticolinérgica. Considerações finais: Evidencia-se que medicamentos como, ansiolíticos e indutores do sono, devem ser prescritos com cautela pelos médicos, visando minimizar efeitos adversos que podem ser causados por esses fármacos quando utilizados a longo prazo.

Palavras-chave: Idoso, Polifarmácia, Ansiolíticos.

\begin{abstract}
Objective: To describe the risks of using anxiolytics and sleep inducers in the elderly population. Bibliographic review: Polypharmacy grows more and more within the elderly population, in view of this, the natural aging process itself generates changes in the pharmacokinetic and pharmacodynamic stages of drugs, bringing some adverse reactions to the elderly. The most used drugs within this age group are represented by the class of anxiolytics and sleep inducers. Long-term use of benzodiazepines whose consumption reaches $15 \%$ to $43 \%$ in adults aged 65 years and over increases the risk of side effects, such as dependence, sedation, cognitive and psychomotor impairment and falls. The sleep inducers, mainly represented by tricyclic antidepressants, can generate sedation, postural hypotension, constipation and dementia effects in the elderly, due to their high anticholinergic action. Final considerations: It is evident that medications such as anxiolytics and sleep inducers should be carefully prescribed by physicians, with few adverse effects that can be caused by these drugs when used in the long term.
\end{abstract}

Key words: Elderly, Polypharmacy, Anxiolytics.

${ }^{1}$ Faculdade de Ensino Superior da Amazônia Reunida (FESAR), Redenção - PA.

*E-mail: fr29gabrielle@gmail.com

2 Universidade de Rio Verde (UniRV), Goianésia - GO.

${ }^{3}$ Faculdade de Minas (FAMINAS-BH), Belo Horizonte - MG.

${ }^{4}$ Faculdade Brasileira Multivix (MULTIVIX), Vitória - ES.

${ }^{5}$ Faculdade de Ciências Médicas e da Saúde de Juiz de Fora (SUPREMA), Juiz de Fora - MG.

${ }^{6}$ Centro Universitário Faminas (FAMINAS), Muriaé - MG.

${ }^{7}$ Universidade José do Rosário Vellano (UNIFENAS), Alfenas - MG.

8 Universidade Vila Velha (UVV), Vila Velha - ES.

${ }^{9}$ Centro Universitário de Caratinga (UNEC), Caratinga - MG. 


\section{RESUMEN}

Objetivo: Describir los riesgos del uso de ansiolíticos e inductores del sueño en la población anciana. Revisión bibliográfica: La polifarmacia crece cada vez más en la población anciana, por lo que el propio proceso de envejecimiento natural genera cambios en las etapas farmacocinéticas y farmacodinámicas de los fármacos, trayendo algunas reacciones adversas a las personas mayores. Los medicamentos más utilizados dentro de este grupo de edad están representados por la clase de ansiolíticos e inductores del sueño. El uso prolongado de benzodiazepinas cuyo consumo alcanza del $15 \%$ al $43 \%$ en adultos de 65 años y más aumenta el riesgo de efectos secundarios, como dependencia, sedación, deterioro cognitivo y psicomotor y caídas. Los inductores del sueño, representados principalmente por antidepresivos tricíclicos, pueden generar efectos de sedación, hipotensión postural, estreñimiento y demencia en los ancianos, debido a su alta acción anticolinérgica. Consideraciones finales: Es evidente que los médicos deben recetar cuidadosamente medicamentos como los ansiolíticos y los inductores del sueño, con pocos efectos adversos que pueden causar estos medicamentos cuando se usan a largo plazo.

Palabras clave: Anciano, Polifarmacia, Ansiolíticos.

\section{INTRODUÇÃO}

A abordagem do paciente idoso na prática clínica é desafiadora e muito particular. Sabe-se que as doenças mais comuns atendidas no dia a dia dos pronto-atendimentos podem se manifestar nos idosos com sinais e sintomas atípicos que, se não percebidos corretamente, podem dificultar o diagnóstico e o manejo correto do paciente. Assim também ocorre com as drogas administradas à essa população. Vale ressaltar que, devido à quantidade de comorbidades que envolvem a senilidade, há ainda tendência à polifarmácia, o que dificulta ainda mais a elucidação dos casos (FARIAS AD, et al., 2021).

A polifarmácia, segundo a Organização Mundial de Saúde (OMS), é o uso de 4 medicamentos ou mais, diariamente, por um paciente. Os medicamentos mais utilizados pelos pacientes idosos são hipotensores, diuréticos, AAS, ansiolíticos, antidepressivos, hipoglicemiantes e analgésicos (OLIVEIRA PC, et al., 2021).

O uso em demasia de antidepressivos e ansiolíticos pelos idosos merece destaque por serem medicamentos com muitos efeitos colaterais e por se tratar de uma parcela da população já tão frágil. Os mecanismos de ação devem ser conhecidos bem como suas indicações, além da observação da adaptabilidade do idoso aos medicamentos prescritos (MORENO CRC, et al., 2019).

Os mecanismos de ação dos antidepressivos são diversos e subdivididos em classes, as mais utilizadas na prática clínica, atuam aumentando o tempo da ação e a quantidade de serotonina, neurotransmissor estimulador, disponível no Sistema Nervoso Central (SNC) (SABELLA D, et al., 2018). Os ansiolíticos, com destaque para os benzodiazepínicos, atuam facilitando a ação do Ácido Gama-Aminobutírico (GABA), consequentemente, diminuindo o fluxo de transmissão no Sistema Nervoso Central (SNC), por isso, são muito utilizados como indutores do sono (GONÇALVES OHP, et al., 2020).

O uso desses medicamentos nos idosos também pode decorrer dos sintomas comuns do processo de envelhecimento, como: inversão do ciclo circadiano, diminuição das sinapses nervosas e perda da massa cinzenta (BUSHATSKY A, et al., 2019). Essas e outras alterações afetam a interação social, podendo desencadear crises de identidade, diminuição da interação com outras pessoas, perda da personalidade e depressão (SOUZA GA, et al., 2020).

Os principais efeitos colaterais dos benzodiazepínicos são: comprometimento cognitivo, delirium e instabilidade postural e dos antidepressivos tricíclicos são: sedação e hipotensão, com isso, um dos maiores riscos que se pode observar com o uso desses medicamentos são as quedas ortostática (ALVIM MM, et al., 2017; OLIVEIRA MG, et al., 2016).

Mundialmente, esse evento é mais observado em pacientes maiores de 65 anos, aumentando conforme a idade pois somam com as alterações fisiológicas do envelhecimento, como: doença aguda, alteração da glicemia, anemia e artrite (SOUSA LMM, et al., 2016). Os fatores ambientais também ganham relevância nessa idade (DAVIES EA, et al., 2015). 
As quedas podem resultar em lesões graves ou fraturas, que necessitam de atenção médica especializada. A prevenção dessas lesões é essencial, visto que os riscos de mortalidade e morbidade são elevados, além de causar mais internações (OLIVEIRA MG, et al., 2016). O presente artigo teve como objetivo realizar uma revisão da literatura a fim de elucidar os medicamentos que mais são utilizados nos idosos, dando destaque aos riscos do uso de ansiolíticos e indutores do sono nesses pacientes.

\section{REVISÃO BIBLIOGRÁFICA}

\section{Polifarmácia}

No Critério de Beers, os medicamentos potencialmente inadequados ou grupos farmacológicos mais utilizados foram: Benzodiazepínicos, Nifedipino, Anti-inflamatórios Não Esteróides (AINES), antidepressivos tricíclicos, entre outros. Em relação aos antidepressivos tricíclicos (Amitriptilina, Nortriptilina, Imipramina), o efeito colinérgico nos idosos tende a ser exacerbado, considerando-os inadequados para tal faixa etária. Já aos benzodiazepínicos (Alprazolam, Clonazepam, Diazepam), os idosos apresentam sensibilidade elevada para tal classe e redução do seu metabolismo, desenvolvendo diversos eventos adversos (AMERICAN GERIATRICS SOCIETY (AGS), 2019).

As Doenças Crônicas Não Transmissíveis (DCNT) têm se tornado as principais causas de morbidade e mortalidade no Brasil, tendo em vista o processo de envelhecimento da população brasileira e a transição epidemiológica que o país vem sofrendo. Essa parte crescente da população tende a apresentar poli morbidade e, consequentemente, tomam muitos medicamentos, configurando a polifarmácia. Diante da poli morbidade, a prevalência da polifarmácia é de 3\%,13\%,37\%,60\% em idosos com uma, duas, três e quatro ou mais DCNT, respectivamente (RAMOS LR, et al. 2016).

Sendo assim, uso racional dos medicamentos é fundamental a fim de garantir o tratamento adequado aos idosos e prevenir potenciais eventos adversos devido às alterações fisiológicas do envelhecimento, que repercutem com mudanças no perfil farmacocinético e farmacodinâmico de inúmeros medicamentos (ANACLETO TA, 2017; RAMOS LR, et al., 2016).

No estudo Fibra, de acordo com Marques PP, et al. (2019), a polifarmácia teve como prevalência 18,4\%, sendo reduzida nos grupos que interpretaram sua saúde como muito boa/boa, que não possuíam assistência privada à saúde e nos não brancos. Ademais, houve associação positiva à polifarmácia, quando presença de obesidade, circunferência da cintura muito aumentada e presença de duas ou três e mais DCNT. Cerca de 20,3\% dos idosos tiveram acesso aos medicamentos via Unidade Básica de Saúde e 13,5\% adquiriram por meios próprios. (MARQUES PP, et al., 2019).

$\mathrm{Na}$ Pesquisa Nacional de Acesso, Utilização e Promoção do Uso Racional de Medicamentos (PNAUM), a prevalência da polifarmácia nos idosos foi de $18 \%$, sendo maior entre os idosos com 70 a 79 anos (20\%), nos obesos $(25,4 \%)$, na região Sul $(25 \%)$, nos que têm plano de saúde $(22,2 \%)$ e nos que autoavaliaram sua saúde como ruim/muito ruim (36,8\%). Em geral, 17,0\%, 17,0\%, 21,0\%, 14,0\% dos idosos relataram utilizar um, dois, três a quatro e pelo menos cinco medicamentos, respectivamente. E há aumento da prevalência em relação à morbidade, sendo de $3 \%$ nos idosos com uma doença, de $13 \%$ naqueles com duas doenças, de $37 \%$ nos com três doenças e $60 \%$ nos com quatro ou mais doenças. Sendo assim, infere-se que com o aumento da idade, dos recursos e das DNTC por pessoa, a polimedicação aumenta (RAMOS LR, et al. 2016).

Portanto, é essencial a identificação desses subgrupos de idosos com maior propensão à polifarmácia para que prescrições e ações individualizadas sejam empregadas adequadamente, visando um uso racional dos medicamentos, proporcionando resultado terapêutico eficaz e qualidade de vida e minimizando eventos indesejáveis e adversos (RAMOS LR, et al. 2016; MARQUES PP, et al., 2019).

\section{Justificativas para o uso dos ansiolíticos e indutores do sono}

Os Benzodiazepínicos (BZD) constituem uma classe terapêutica de ansiolíticos amplamente consumida pela população em geral, sendo que, segundo Victorri-Vigneau C, et al. (2020), o consumo de BZD pelos adultos com 65 anos ou mais alcança uma taxa de 15\% a 43\%. Esses medicamentos são utilizados por essa 
faixa etária com fins de controle e prevenção de ansiedade, insônia e transtornos comportamentais e de humor (ALVIM MM, et al., 2017).

Contudo, tais remédios apresentam alto risco de efeitos colaterais, como dependência física e psicológica, maior frequência de quedas e eventos cognitivos e psicomotores adversos, especialmente quando se adota o uso de forma crônica, ao longo de meses ou anos (ALVARENGA JM, et al., 2015). Diante disso, os benzodiazepínicos foram incluídos na lista de Medicamentos Potencialmente Inapropriados para Idosos (MPI), principalmente os de longa duração (MOREIRA FSM, et al., 2020).

Apesar dos riscos, os quais em diversas situações são irreconhecíveis, subestimados ou negados pelos idosos, esses adotam o uso dos BZD baseados em inúmeras justificativas: dificuldade para dormir e para lidar com as situações cotidianas; sintomas de labirintite; nervosismo; solidão; sintomas depressivos; medo da velhice; transtornos psiquiátricos; problemas financeiros; preocupação com os membros da família e reação ao luto (ALVARENGA JM, et al., 2015). Somado a isso, segundo Alvim MM, et al. (2017), a minimização da tolerância ao estresse, juntamente com a apresentação e a propaganda de novas drogas e a prescrição imprópria pelos profissionais contribuem para uma crescente utilização dos benzodiazepínicos.

Isso demonstra, portanto, que fatores sociais, interpessoais, culturais e os envolvidos com o desenvolvimento do indivíduo contribuem para o uso do BZD de maneira contínua e irracional, fora das indicações médicas adequadamente controladas. Ademais, há a colaboração de outros fatores, como a busca dos idosos pelo alívio e esquecimento, receio do retorno e intensificação dos sintomas, subestimação ou negação dos efeitos adversos, escassez de vigilância e aconselhamento/acompanhamento médico, além da facilidade na aquisição de prescrição médica sem necessidade da consulta (ALVARENGA JM, et al., 2015; ALVIM MM, et al., 2017).

Tratando-se dos antidepressivos, que são os psicotrópicos comumente associados aos benzodiazepínicos, seu consumo tem crescido no mundo inteiro, especialmente entre os idosos, do sexo feminino, que são casadas. Essa associação medicamentosa é benéfica no início do tratamento da depressão em virtude dos sintomas de ansiedade e insônia. Contudo, o uso prolongado é contraindicado devido aos efeitos colaterais (NALOTO DC, et al., 2016).

Existem variadas classes de antidepressivos, sendo que um medicamento bastante utilizado por essa faixa etária é a amitriptilina, da classe dos antidepressivos tricíclicos, a qual é usada com o intuito de melhorar o humor, minimizar a ansiedade e sintomas não específicos de sofrimento psicológico, induzir ao sono, tratar a dor e a depressão. Entretanto, a amitriptilina faz parte dos MPI, haja vista que é altamente anticolinérgica, sedativa e causadora de hipotensão ortostática. Sendo assim, é necessário realizar uma análise do riscobenefício e da possibilidade de fármacos alternativos (OLIVEIRA MG, et al., 2016).

\section{Mecanismos farmacológicos e principais riscos associados ao uso de benzodiazepínicos e antidepressivos tricíclicos por idosos}

Diversos estudos abordam sobre os efeitos adversos relacionadas ao uso de Benzodiazepínicos (BZD), que são prescritos para transtornos comportamentais, indução do sono e como ansiolítico, sendo responsáveis por causar dependência farmacológica, limitações física e cognitiva, sendo que sua utilização abrangente acarreta riscos entre os idosos (MOREIRA FSM et al., 2020).

Isso ocorre devido ao seu mecanismo de ação, pelo fato de os BZDs atuarem em todo Sistema Nervoso Central (SNC) como drogas psicotrópicas, por meio da transmissão sináptica inibitória ao modular o receptor subtipo A do Ácido Gama-Aminobutírico (GABA A). Ademais, também exercem efeito em um ponto regulador específico do GABA A, acentuam o efeito inibitório do neurotransmissor e causam efeito depressor do SNC (FARIA JSS, et al., 2019).

Além disso, sua relação tolerância/dependência é baseada na farmacocinética, alta lipossolubilidade e meia vida biológica. Tais fatores se encontram alterados nos idosos, devido a idade, diminuição do tônus muscular, da taxa de filtração glomerular e da excreção renal, assim como alterações no metabolismo hepático sobre a biodisponibilidade do fármaco (MOREIRA P e BORJA A, 2018). 
Segundo Alvarenga JM, et al. (2015) em seu estudo de base populacional, foi evidenciado que o uso prolongado de BZD por idosos, caracterizado a partir de 6 meses, está relacionado principalmente a efeitos adversos cognitivos e psicomotores, que podem causar quedas e fraturas, devido aos efeitos sedativos residuais, delirium e a instabilidade postural (MOREIRA FSM, et al., 2020; OLIVEIRA MG, et al., 2016). Também são relatados como reações adversas, a presença de amnésia, ataxia e a intensificação do efeito depressor em interação com outras drogas depressoras do SNC (ALVIM MM, et al., 2017). Além disso, estudos de Hosseinzadeh ZB, et al. (2021), apontam disfunções sexuais ligadas ao uso de BZDs, como a diminuição da libido e a disfunção erétil.

Em casos de superdosagem e dependência, podem causar sono prolongado, devido ao aumento do sono REM, com disfunção grave das funções respiratória e cardiovascular, além da Síndrome de privação, principalmente nos BZDs de ação de curta duração, que com a sua retirada abrupta provocam efeito rebote da ansiedade durante semanas, associado a presença de tonturas, tremores, zumbidos e emagrecimento (RANG HP, et al., 2016).

Os idosos realizam o uso simultâneo com antidepressivos, em especial os de segunda geração, tricíclicos, como a amitriptilina e nortriptilina. Essa classe farmacológica atua por meio da inibição da recaptação de monoaminas pelas terminações nervosas, competindo pelo sítio de ligação no transportador de aminas e inibe também a recaptação de norepinefrina e de 5-HT, o que leva ao alívio dos sintomas biológicos da depressão, devido a facilitação de transmissão noradrenérgica (RANG HP, et al., 2016).

No que tange aos antidepressivos, também são encontrados efeitos adversos psicomotores, devido a interferência no controle autônomo e a ação anticolinérgica pronunciada, levando a sedação, demência, hipotensão postural, boca seca, constipação e aumento do apetite (ANACLETO TA, 2017). Além disso, em superdosagem acarreta vários riscos devido a sua grande interação com as bebidas alcoólicas e outros fármacos, por exemplo: os anestésicos, como a cetamina em doses elevadas e os anti-hipertensivos, em especial o metoprolol, o que provoca excitação do SNC e do Sistema Cardiovascular, levando ao delirium, arritmias e convulsões (RANG HP, et al., 2016).

\section{Riscos de Quedas}

Segundo Coutinho ESF e Silva SD (2002) as quedas são categorizadas como problema de saúde pública entre a população idosa, tendo vista que as fraturas resultantes destas exigem cuidados especializados, os quais possuem elevado custo. Portanto, de acordo com Sousa LMM, et al. (2016), devem ser evitadas por meio da minimização dos fatores multifatoriais que ocasionam a queda, como organização do ambiente, ajustes dos agentes farmacológicos, dentre outros.

Autores como Davies EA, et al. (2015), relatam que o envelhecimento tem como resultado diversas mudanças fisiológicas, bem como alterações farmacodinâmicas e farmacocinéticas das drogas que elevam as chances de eventos adversos, pois aumentam o tempo de meia-vida dos medicamentos e, ainda a necessidade da combinação farmacológica, devido à concomitância de doenças crônicas o que potencializa os episódios prejudiciais. Coutinho ESF e Silva SD (2002) apontam que a associação de bloqueadores de canais de cálcio e benzodiazepínicos ampliam os riscos dos acidentes seguidos de fraturas.

Para tanto, estima-se que a polifarmácia é umas das maiores responsáveis pelo aumento do risco de quedas entre os idosos, principalmente quando é incluído a associação de Medicamentos Potencialmente Inapropriados (MPI), dentre os quais se destaca a prescrição de benzodiazepínicos e antidepressivos, que possuem alta taxa residual, acarretando aumento da ocorrência de quedas, já que possuem efeitos sedativos e de depressão do SNC (MOREIRA FSM, et al., 2020).

Os Critérios de Beers, desenvolvidos em 1991 e atualizados em 2015, classificam os fármacos de acordo com o mecanismo de atuação, interação, além de classificá-los em grupos farmacológicos que devem ser evitados pelos idosos e os fármacos que necessitam ser utilizados com cautela. Logo, os benzodiazepínicos, como Alprazolam, Bromazepam, e Diazepam, foram incluídos na classe de drogas que precisam ser evitadas em idosos, pois criam a susceptibilidade a Delirium, comprometimento cognitivo, quedas e outros acidentes (OLIVEIRA MG, et al., 2016). 


\section{Formas de prevenir polifarmácia e reduzir quedas}

Um conjunto de condições para determinar o uso de medicamentos que não deveriam ser utilizados por idosos em asilos nos Estados Unidos foi determinado a partir de 1991 por Beers. Tal trabalho envolveu um grupo de especialistas multidisciplinares, utilizando a técnica Delphi. A técnica basicamente consiste em obter informações a partir de questionários respondidos pela própria população em estudo, as respostas são avaliadas criando assim um novo questionário e assim por diante, até obter um resultado que evidencie acordo entre os participantes. O principal objetivo de um consenso sobre o assunto que ainda está sob investigação (ROCHA A, et al., 2020).

A atualização dos critérios de Beers é realizada a cada 3 anos pela AGS. Concentrados nas categorias de ansiolíticos, antiagregantes plaquetários, antialérgicos, antianginosos e vasodilatadores, antiulcerosos, antidepressivos, anti-hipertensivos, antiespasmódicos, anti-inflamatórios não esteroidais, antiarrítmicos e glicosídeos cardíacos. A última alteração ocorreu em 2019, não indicando a prescrição de mais 30 medicamentos e outros 40 medicamentos que devem ser usados com cautela (AGS, 2019). Com a nova atualização assegura-se o uso e a eficaz dos medicamentos pelos pacientes idosos e consequentemente tratamento mais seguro (COMELATO C e SERRANO PG, 2019).

Em relação ao risco de queda, projetos de exercícios com duração de 10 semanas a 9 meses aplicados em idosos obtém uma redução em $10 \%$ da probabilidade de queda comparado aos idosos sedentários. Além disso, treinamentos específicos para o equilíbrio, resultaram em uma redução de $25 \%$ de quedas. Segundo Marinho MS, et al. (2019), aulas específicas de exercício de equilíbrio, como o Tai Chi Chuan, representam uma redução de até $47 \%$ dos riscos.

\section{CONSIDERAÇÕES FINAIS}

Sabe-se que a polifarmácia nos idosos vem sendo um desafio constante na conduta médica, principalmente quando é abordado o risco-benefício do uso de medicações. Logo, estudos demonstram que Benzodiazepínicos e Antidepressivos tricíclicos são os mais associados, provocando eventos psicomotores e cognitivos adversos, aumentando o risco de quedas e a dependência medicamentosa. É válido ressaltar a cautela na prescrição de fármacos à população idosa, tendo uma visão que abranja possíveis interações medicamentosas, experiência cotidiana, comorbidades presentes e os riscos de uso a longo prazo. Importante evidenciar a singularidade de cada paciente bem como o seu tratamento, aprimorando a conduta médica e sendo mais prudente ao medicar, dando relevância aos critérios que minimizem os efeitos adversos deles.

\section{REFERÊNCIAS}

1. AMERICAN GERIATRICS SOCIETY (AGS). American Geriatrics Society Beers Criteria Update Expert Panel. American Geriatrics Society 2019 Updated AGS Beers Criteria® for Potentially Inappropriate Medication Use in Older Adults. J Am Geriatr Soc, 2019; 67(4): 674-694.

2. ALVARENGA JM, et al. Uso de benzodiazepínicos entre idosos: o alívio de "jogar água no fogo", não pensar e dormir. Revista Brasileira de Geriatria e Gerontologia, 2015; 18(2): 249-258.

3. ALVIM MM, et al. Prevalence of and factors associated with benzodiazepine use in community-resident elderly persons. Revista Brasileira de Geriatria e Gerontologia, 2017; 20: 463-473.

4. ANACLETO TA. Medicamentos potencialmente inadequados para idosos. Boletim ISMP, 2017;7(3).

5. BUSHATSKY A, et al. Fatores associados às alterações de equilíbrio em idosos residentes no município de São Paulo em 2006: evidências do Estudo Saúde, Bem-Estar e Envelhecimento (SABE). Revista Brasileira de Epidemiologia. 2019, v. 21.

6. COMELATO C, SERRANO PG. Atualização dos Critérios de Beers AGS 2019, para medicações potencialmente inapropriadas em idosos. 2019.

7. COUTINHO ESF, SILVA SD. Uso de medicamentos como fator de risco para fratura grave decorrente de queda em idosos. Cadernos de Saúde Pública, 2002; 18: 1359-1366.

8. DAVIES EA, et al. Adverse drug reactions in special populations - the elderly. British journal of clinical pharmacology, 2015; 80: 796-807.

9. FARIA JSS, et al. Benzodiazepínicos: revendo o uso para o desuso / Benzodiazepines: reviewing the use for disuse. Rev Med (São Paulo), 2019; 98(6): 423-6. 
10. FARIAS AD, et al. Prescrição de medicamentos potencialmente inapropriados para idosos: um estudo na Atenção Primária à Saúde. Ciência \& Saúde Coletiva, 2021; 26: 1781-1792.

11. GONÇALVES OHP, et al. Benzodiazepinics and the treatment of delirium: a literature review. Revista da Associação Médica Brasileira, 2020; 66: 998-1001.

12. HOSSEINZADEH ZB, et al. Benzodiazepines related sexual dysfunctions: A critical review on pharmacology and mechanism of action. Rev Int Androl, 2021; 19(1): 62-68.

13. MARINHO MS, et al. Efeitos do Tai Chi Chuan na incidência de quedas, no medo de cair e no equilíbrio em idosos: uma revisão sistemática de ensaios clínicos aleatorizados. Revista Brasileira de Geriatria e Gerontologia, 2019, 10: 243-256.

14. MARQUES PP, et al. Polypharmacy in community-based older adults: results of the Fibra study. Rev. bras. geriatr. Gerontol, 2019; 22(5): e190118.

15. MOREIRA FSM, et al. Uso de medicamentos potencialmente inapropriados em idosos institucionalizados: prevalência e fatores associados. Ciência \& Saúde Coletiva, 2020; 25 (6): 2073-2082.

16. MOREIRA P, BORJA, A. Benzodiazepínicos: uso e abuso em pacientes idosos. Centro de Pós-Graduação, Pesquisa e Extensão Oswaldo Cruz, 2018.

17. MORENO CRC, et al. Problemas de sono em idosos estão associados a sexo feminino, dor e incontinência urinária. Revista Brasileira de Epidemiologia, 2019; 21.

18. NALOTO DC, et al. Prescrição de benzodiazepínicos para adultos e idosos de um ambulatório de saúde mental. Ciência \& Saúde Coletiva, 2016; 21(4): 1267-1276.

19. OLIVEIRA PC, et al. Prevalência e Fatores Associados à Polifarmácia em Idosos Atendidos na Atenção Primária à Saúde em Belo Horizonte - MG, Brasil. Ciência \& Saúde Coletiva, 2021; 26: 1553-1564

20. OLIVEIRA MG, et al. Brazilian consensus of potentially inappropriate medication for elderly people. Geriatr Gerontol Aging, 2016; 10: 168-181.

21. RAMOS LR, et al. Polifarmácia e polimorbidade em idosos no Brasil: um desafio em saúde pública. Rev Saúde Pública, 2016; 50(supl 2): 1-9.

22. RANG HP, et al. Farmacologia 8. ed. Rio de Janeiro: Elsevier, 2016: 1251-1312

23. ROCHA A, et.al. Evolução histórica do uso de medicamentos potencialmente inadequados: Critérios de Beers em 10 anos. Brazilian Journal of Development, 2020; 6(6): 36178-36191.

24. SABELLA D. Antidepressant Medications. The American journal of nursing, 2018; 118(9): 52-59.

25. SOUSA LMM, et al. Risco de quedas em idosos residentes na comunidade: revisão sistemática da literatura. Revista Gaúcha de Enfermagem, 2016; 37: e55030.

26. SOUZA GA, et al. Percepções de pessoas idosas sobre o cuidado com o(a) idoso(a) frágil na comunidade, 2020.

27. VICTORRI-VIGNEAU C, et al. Are Seniors Dependent on Benzodiazepines? A National Clinical Survey of Substance Use Disorder. Clinical Pharmacology \& Therapeutics, 2020; 0: 1-8. 